\title{
Estimation of Mobility Models Based on Mobility Metrics and Their Impact on Routing Protocols in MANET
}

\author{
S. C. Sharma and Santosh Kumar
}

\begin{abstract}
Mobile adhoc network (MANET) routing protocols performance are perceptive to mobility and scalability of network, therefore, the objectives of paper is to describe mobility metrics into direct and derived mobility metrics and impact of these metrics on routing performance metrics in MANET. An effort for analyzing derived mobility metrics with direct mobility metrics are considered across Manhattan and Freeway mobility model in this article. This article extends an intuitive study to analyze impact of mobility models on two prominent reactive routing protocols i.e. ad-hoc on demand distance vector (AODV) and dynamic source routing (DSR) with fixed network size, varying node speed and identical traffic load.
\end{abstract}

Index Terms-Mobility models, mobility metrics, connectivity graph metrics, routing protocol-AODV, DSR.

\section{INTRODUCTION}

Manhattan mobility model (MMM) predicts mobility patterns of mobile nodes on bidirectional street and useful in modeling of urban area vehicular traffic [1], [2]. It is composed of horizontal and vertical street map (North and South direction of vertical streets, East and West for horizontal streets), which causes geographical restriction of network. The mobile node is allowed to move along a grid of horizontal and vertical streets on the map. At an intersection of a horizontal and a vertical street, the mobile node can turn left, right or go straight on a probability basis. The velocity of a mobile node at a time slot is dependent on its velocity at previous time slot. Also, a node's velocity is restricted by the velocity of node preceding it on the same lane of the street. Inter-node and intra-node relationships involved same as in Freeway model. Thus, the Manhattan mobility model realizes spatial and high temporal dependencies. Manhattan mobility model are useful for vehicular traffic modeling but inefficient for all aspects of tactical scenario.

Freeway Mobility model (FMM) includes spatial, high temporal dependencies and also impose strict geographical restriction [1]. Mobile node velocity is temporally dependent on its previous velocity. The velocity of a mobile node influenced with other mobile node moving in same lane inside a certain radius (spatial dependence). All mobile node movement is imposed strict geographical restriction. Each mobile node is restricted to its lane on the freeway and velocity of the mobile node is temporally dependent on its

Manuscript received December 5, 2012; revised March 22, 2013.

S. C. Sharma is with the Indian Institute of Technology, Roorkee India (e-mail: scs60fpt@iitr.ernet.in).

Santosh Kumar is with the Graphic Era University, Dehradun, India (e-mail: amu.santosh@gmail.com). previous velocity. If two mobile nodes on the same freeway lane are within safety distance (SD), velocity of the follow node cannot exceed the velocity of preceding nodes.

\section{MOBILITy Metrics}

Mobility metrics were first introduced by P. Johansson et al. [3]. To differentiate various mobility patterns and these mobility pattern Qunwei Zheng et al. [4] classify mobility metrics in two categories: direct and derived metrics. First evaluates the phenomena of clear physical correspondence (such as speed or acceleration) like the temporal dependence, spatial dependence and geographic restrictions. In addition to these metrics, the relative speed metric that differentiates mobility patterns based on relative motion. Second uses mathematical modeling to measure the change to some logical structure (e.g. connectivity graph). The other metrics which is included in this paper is routing performance metrics. These metrics are used to analyse the impact of mobility on routing protocols performance metrics in MANET. The metrics classification is visualized broadly into two categories as:

Mobility metrics

Protocol performance metrics

Further, mobility metrics is categorized into direct and derived mobility metrics. The detailed analysis of direct and derived mobility metrics are discussed and analysed in this paper.

\section{A. Direct Mobility Metrics}

Random Based: Characteristics of this metrics have no dependencies and restriction. This metrics has statistical model, in this node can move to any destination and their velocities and directions are chosen randomly. In this metrics, models are basically idealistic rather than realistic, because in a real world, nodes move randomly without any specified destination.

Relative Speed $(R S)$ : It is standard definition drawn from physics which is based on relative speed [5], [6] of all pairs of nodes in networks over time $t$ i.e. the speed of first node $i$, relative to the second node $j$.

$$
R S(i, j, t)=\left|\vec{V}_{i}(t)-\vec{V}_{j}(t)\right|
$$

Average Relative Speed: Average relative speed $R S(i, j)$ of hosts $i, j$ at time $t$ will be-

$$
\overline{R S}=\frac{\sum_{i=1}^{N} \sum_{j=1}^{N} \sum_{t=1}^{T} R S(i, j, t)}{P}
$$

where $P$ is the number of tuples $(i, j, t)$ such that $\operatorname{RS}(i, j, t) \neq 0$. 
Degree of Temporal Dependence: The temporal dependencies imply how an individual node changes its velocity with respect to time or a node actual movement influenced with its past movement. For each node, it is defined as a product of relative direction and relative speed (relative to its past itself) i.e.

$$
D_{\text {temp }}\left(i, t, t^{\prime}\right)=R D\left(\vec{v}_{i}(t), \vec{v}_{i}\left(t^{\prime}\right)\right) \times S R\left(\vec{v}_{i}(t), \vec{v}_{i}\left(t^{\prime}\right)\right)
$$

The value of $\mathrm{D}_{\text {temp }}\left(i, t, t^{\prime}\right)$ is high when node moves in the same direction and almost at similar speed and decreases if relative direction or the speed ratio decreases over a certain time interval.

$$
\left|t-t^{\prime}\right|>c \Rightarrow D_{\text {temp }}\left(i, t, t^{\prime}\right)=0
$$

where $c>0$ is a constant

Average Degree of Temporal Dependence: The average degree of temporal dependence $D_{\text {temp }}\left(i, t, t^{\prime}\right)$ is the value of averaged over nodes and time instants i.e.

$$
D_{\text {temp }}=\frac{\sum_{i=1}^{N} \sum_{t=1}^{T} \sum_{t^{\prime}=1}^{T} D_{t e m p}\left(i, j, t^{\prime}\right)}{P}
$$

where $P$ is the number of tuples $\left(i, t, t^{\prime}\right)$ such that $\mathrm{D}_{\text {temporal }}(i, t$, $\left.t^{\prime}\right) \neq 0$. It has two conditions that is obvious, first, if the present velocity of a node is fully independent of its velocity at previous time period, then the mobility pattern is expected to have a smaller value for $\mathrm{D}_{\text {temporal }}$. Second, if the current velocity is strongly dependent on the velocity at some previous time step, then the mobility pattern is expected to have a higher value for $\mathrm{D}_{\text {temporal }}$.

Degree of Spatial Dependence: The degree of spatial dependence is a measure of a node's correlation with others nodes in the networks. The degree of spatial dependence between nodes $i, j$, at time $\mathrm{t}\left(\mathrm{D}_{\text {spatial }}(i, j, t)\right)$ by equation 5 .

$$
D_{\text {spatial }}(i, j, t)=R D\left(\vec{v}_{i}(t), \vec{v}_{j}(t)\right) \times S R\left(\vec{v}_{i}(t), \vec{v}_{j}(t)\right)
$$

The value of $D_{\text {spatial }}(i, j, t)$ will increases when nodes $i$ and $j$ move in same direction with almost similar speed and decreases when nodes $i$ and $j$ move in relative direction or dissimilar speed over certain time interval. The spatially dependent on a faraway node will be zero and satisfy following conditions i.e.

$$
D_{i, j}(t)>c \times R \Rightarrow D_{\text {spatial }}(i, j, t)=0
$$

where $c>0$ is a constant.

Average Degree of Spatial Dependence: The average degree of spatial dependence is an average of degree of spatial dependence of all nodes pairs in the network [5] i.e.

$$
D_{\text {spatial }}=\frac{\sum_{t=1}^{T} \sum_{i=1}^{N} \sum_{j=i+1}^{N} D_{\text {spatial }}(i, j, t)}{P}
$$

where $P$ is the number of tuples $(i, j, t)$ such that $\mathrm{D}_{\text {spatial }}(i, j, t)$ $\neq 0$.

\section{1)Evaluation of direct metrics}

Direct metrics evaluation lead for the differentiation of mobility patterns. For this, differentiation a mobility pattern scenario is required which captures the characteristics of relative speed, temporal dependence and spatial dependence.
A user manual for IMPORTANT Mobility Tool Generators in NS-2.34 simulator is used [6].

Simulation Environment Setup- A mobility scenario generator produced different mobility patterns of Manhattan Mobility model (MMM) and Freeway mobility model (FMM). The common scenario parameters for these models are as; transmission range is $250 \mathrm{~m}$, simulation area $1000 \mathrm{~m} \mathrm{x}$ $1000 \mathrm{~m}$, number of nodes are 40, max speed $\left(\mathrm{V}_{\max }\right)$ are $1,5,10$, $20,30,40,50,60 \mathrm{~m} / \mathrm{sec}$ and simulation duration is $900 \mathrm{~s}$. Manhattan mobility model (MMM) minimum allowed velocity and acceleration speed are 0.5 . The simulation setup is created over Fedora 11 (Linux- Platform) environment for direct metrics and derived metrics analysis.

\section{2) Direct metrics result analysis}

Average relative speed: It has been observed from the simulation that the average relative speed of Manhattan mobility model is high than Freeway mobility model.

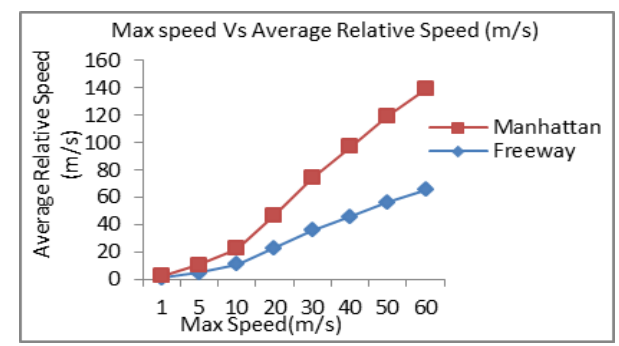

Fig. 1. Max speed $(\mathrm{m} / \mathrm{s})$ Vs average relative speed

Average degree of spatial dependence: The average degree of spatial dependence of Manhattan and Freeway Mobility model is almost zero in present observation shown in Fig. 1.

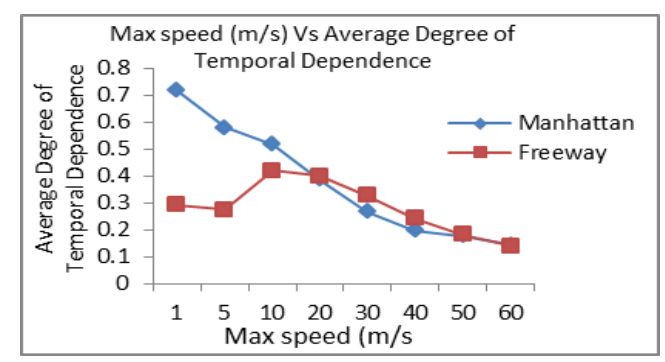

Fig. 2. Max speed (m/s) Vs average degree of temporal dependence

Average degree of temporal dependence: The results analysis of average degree of temporal dependence causes uncertainty for differentiation of different mobility pattern in study but in the present observation, it is observed that as the mobility increases average degree of temporal dependence decreases and Freeway mobility model has low value than Manhattan a little as shown in Fig. 2. The usefulness of this metrics is still in research.

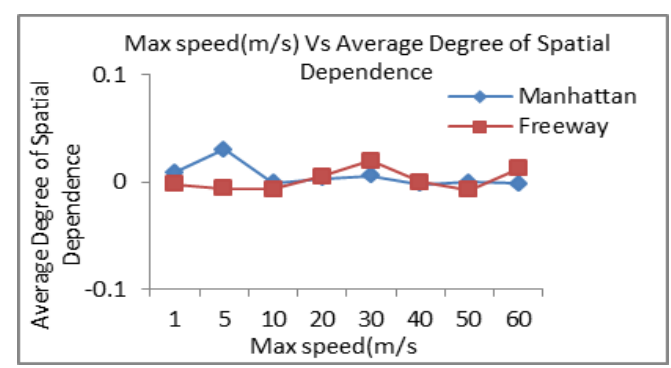

Fig. 3. Max speed $(\mathrm{m} / \mathrm{sec})$ Vs average degree of spatial dependence 


\section{B. Effect Evaluation of Mobility Model on Derived Metrics}

Derived metrics is also known as connectivity graph metrics because it is derived from graph theoretic models as well as other mathematical models. Mobility model impact the connectivity graph which in turn influence the protocol performance. Therefore, it is necessary to study metrics that capture the properties of connectivity graph.

Connectivity Graph: The connectivity graph is the graph $G=(V, E)$ where $|V|=N$, a link $(i, j) \leq E$ iff $D_{i, j}(t) \leq R$. Let $X(i$, $j, t)$ be an indicator random variable which has a value 1 iff there is a link between nodes $i$ and $j$ at time $t$. $X(i, j)=\max _{t=1}^{T} X(i, j, t)$ be an indicator random variable which is 1 if a link existed between nodes $i$ and $j$ at any time during the simulation, 0 otherwise. The graph connectivity metrics includes number of link changes, link duration and path availability.

\section{1)Derived metrics (connectivity graph) result analysis}

Average Number of Link Changes: A link between two hosts is established due to host movement. It is indicator of topology change rate. Link change is total number of link up and downs in unit time. Although, the average number link changes metric is unable to differentiate several mobility patterns even though an effort has been done for Manhattan and Freeway mobility model and found that average number of link change of Freeway mobility model is high than Manhattan mobility model as shown in Fig. 4.

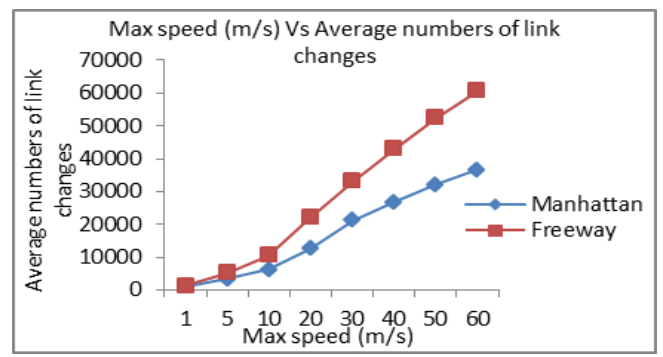

Fig. 4. Max speed (m/s) Vs average numbers of link changes

Average Link Duration: The average link duration for Freeway and Manhattan mobility model get down as the mobility speed increases; it may be because of opposite direction and high relative speed as shown in Fig. 5.

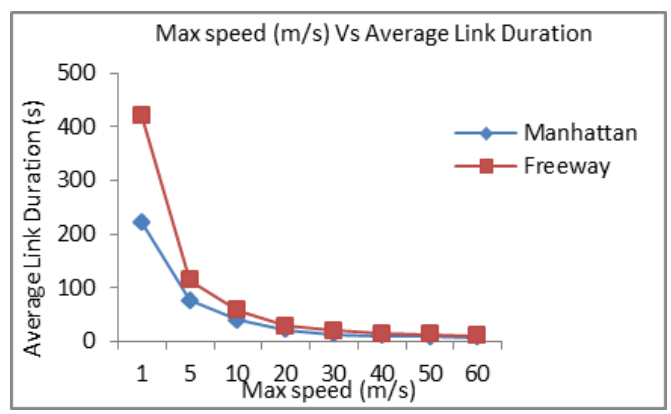

Fig. 5. Max speed $(\mathrm{m} / \mathrm{sec})$ Vs average link duration

\section{IMPACT OF MOBILITY MOdELS ON ROUTING PROTOCOL PERFORMANCE METRICS}

The relationship between mobility metrics and performance metrics were unclear but after introduction of connectivity metrics in section $\mathrm{B}$, it is very clear co-relationship between mobility metrics (i.e. average relative speed, average degree of temporal dependence, and average degree of spatial dependence, average number of link changes, average path duration, average path availability) and performance metrics of the routing protocols i.e. packet delivery fraction, routing overhead and average end-to-end delay.

Simulation Environment Setup- Two mobility models Manhattan, and Freeway are obtained with generating scenario by setting the parameter of mobility model accordingly in terms of fixed network load as well as varying speed by mobility scenario generator tool and considered for performance analysis of two on-demand (AODV, DSR) routing protocols in the present work. NS 2.34 is taken as a specific tool, due to its open source code base and specific protocol IEEE 802.11 b.

The objective of analysis is to observe, how the routing protocols performance affected with different mobility pattern in fixed network size of 40 nodes and varying node speed $1,5,10,29,30,40,50,60(\mathrm{~m} / \mathrm{s})$ with $900 \mathrm{~s}$ simulation time in mobile adhoc environment. A 'cbr' data packet application of size 512 bytes is taken. The simulation is carried out in region of $1000 \mathrm{~m} \times 1000 \mathrm{~m}$ in present analysis.

\section{A. AODV Performance Metrics Analysis}

From Fig. 6 (a) to Fig. 6 (c), it is observed that AODV has higher throughput and lower overhead, lower end-to-end delay in Manhattan, Freeway mobility model respectively.

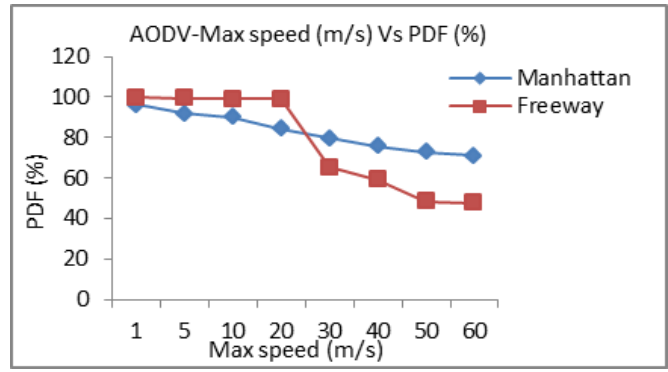

Fig. 6. (a)AODV-max speed (m/sec) Vs PDF (\%)

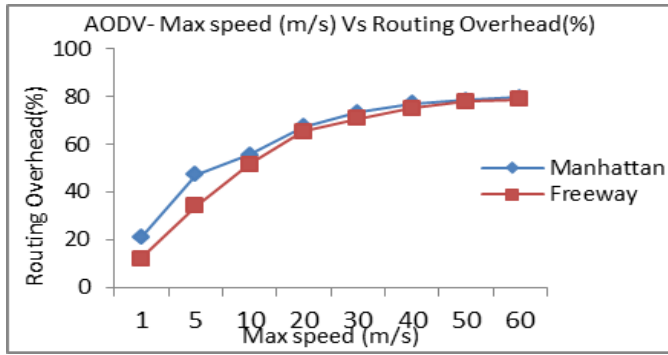

Fig. 6. (b)AODV-max speed (m/s) Vs routing overhead (\%)

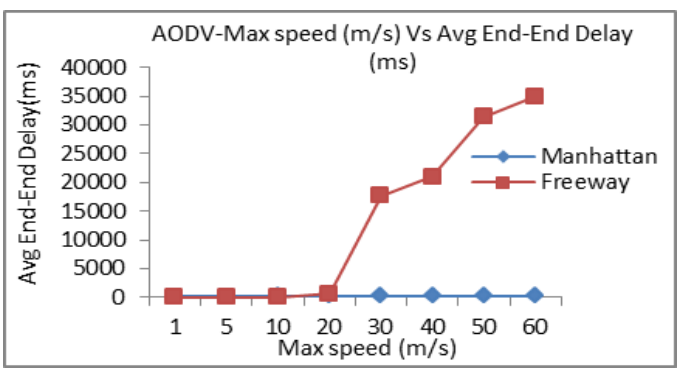

Fig. 6. (c)AODV-max speed (m/s) Vs average end-end delay (ms) 


\section{B. DSR Performance Metrics Analysis}

From Fig. 7 (a) to Fig. 7 (c), it is observed that DSR has higher throughput and lower overhead, lower end-to-end delay in Manhattan, Freeway mobility model respectively.

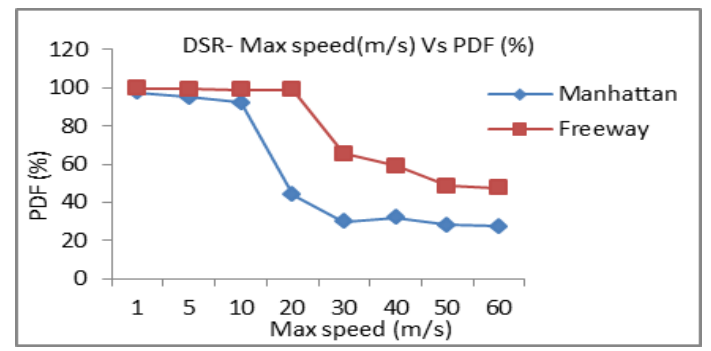

Fig. 7. (a)DSR- max speed (m/s) Vs PDF (\%)

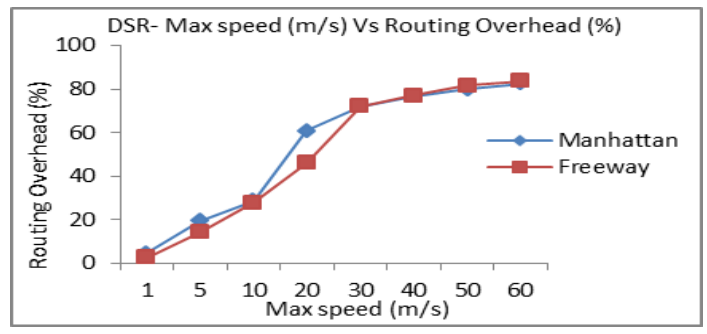

Fig. 7. (b)DSR- max speed ( $\mathrm{m} / \mathrm{sec})$ Vs routing overhead (\%)

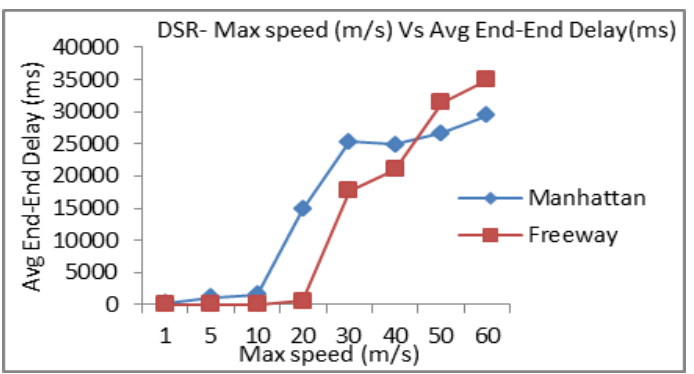

Fig. 7. (c)DSR- max speed (m/s) Vs average end to end delay (ms)

\section{VALIDATION AND CONCLUSION}

For given average relative speed, if the average degree of spatial dependence is high then it signifies that there is a link between two nodes. It indicates that there is a stable link for longer period of time to move together and signifies the link duration is high. If the link between source and destination is stable then drop of the packet will be less which increases throughput of routing protocol. At same time control overhead is lower which require little effort to repair broken path. If the mobility model has high relative speed then nodes might be move out range. This leads more packet drops which results lower throughput. Therefore, higher control overhead needed to repair the broken link. At fixed speed, if same spatial dependence is considered then freeway and Manhattan mobility model have high relative which lead lower link duration shown in Fig. 1, Fig. 3 and Fig. 5. Therefore, lower link duration results lower throughput and higher overhead, end-to-end delay.
From performance metrics (i.e. throughput, routing overhead and end-to-end delay) result analysis, it is observed that there is no protocol which is performing well in every condition for both models taken. Therefore, present analysis concludes that routing protocols performance rely on scenario conditions and mobility model.

\section{REFERENCE}

[1] F. Bai, N. Sadagopan, and A. Helmy, "Important: A framework to systematically analyze the impact of mobility on performance of routing protocols for Adhoc networks," in Proc. IEEE INFOCOM, San Francisco, USA, pp. 825-835, 2003.

[2] User Manual for Important Mobility Scenario Generation and Analysis Tool, University of Bonn, Dec, 2010.

[3] P. Johansson, T. Larsson, N. Hedman, B. Mielczarek, and M. germark, "Scenario-based performance analysis of routing protocols for mobile ad-hoc networks," in Proc. International Conference on Mobile Computing and Networking (MobiCom'99), pp. 195-206.

[4] Q. W. Zheng, X. Y. Hong, and S. Ray, "Recent advances in mobility modeling for mobile Ad Hoc," in ACMSE'04, Huntsville, Alabama, USA Copyright 2004 ACM 1581138709/04/04.

[5] F. Bai, N. Sadagopan, and A. Helmy, User Manual for Important Mobility Tool Generators in NS-2 Simulators, 2004.

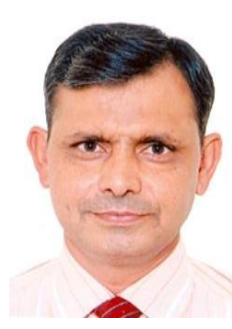

S. C. Sharma received Ph.D. Electronics and Computer Engg.) and M.Tech. (Electronics and Communication Engg.) in 1992 and 1983 respectively from IIT Roorkee (erstwhile University of Roorkee), the honorary member of NSBE, ISOC, and IAENG, ACEEE. He started his career as R \& D Engineer in 1983 then joined teaching profession in Jan. 1984 in IIT-Roorkee and continuing till date. He has published over hundred thirty six research papers in national and international journals/conferences and supervised more than 30 projects/dissertation of PG students. He has supervised several Ph.D. in the area of Computer Networking, Wireless Network, Computer Communication and continuing supervising Ph.D. in the same area. Currently, he is supervising Six Ph.D. Scholars. He has successfully completed several major research projects independently and many minor research projects related to Communication and SAW filter Design sponsored by Government of India. IIT-Roorkee has awarded him the Khosla annual research prize with best research paper in the year 2000 . His many research papers have been awarded by National and International Committees. He has worked as research scientist at FMH, Munchen, Germany in the year 1999. He is the active reviewer of two reputed International Journals and Editor of National Journal (BITS, PILANI). He has also worked as Group leader of Electronics \& Instrumentation Engg. Department of BITS-Pilani-Dubai Campus, from Aug. 2003 to Aug. 2005. Presently he is continuing as Associate Professor at IIT Roorkee-India.

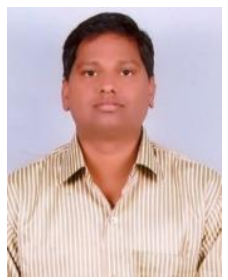

Santosh Kumar received Ph.D. (Electronics and Computer Engineering) from IIT Roorkee, Roorkee (India) in 2012, M.Tech.(Computer Science \& Engineering) from Aligarh Muslim University, Aligarh (India) in 2007 and B.E.( Information Technology) from C.C.S. University, Meerut (India) in 2003. He is member of ACEEE, IAENG, ISOC (USA) and reviewer of International Journal "IJACSA". He has published over seventeen research articles in National \& International Journals/conferences in field of Wireless Communication Networks, Mobile Computing and Grid Computing. He has more than 7.6 years of experience in teaching of UG (B.Tech.) \& PG (M.Tech.) level courses in teaching as a Lecturer/Assistant Professor. Currently, he is working as an assistant professor in Department of Computer Science and Engineering, Graphic Era University, Dehradun (India). 